\title{
Characterization of the fatigue behaviour and lifetime evaluation of metallic materials based on thermographic NDT-methods
}

\author{
by H. Wu***, P. Starke*, C. Boller*
}

\author{
${ }^{*}$ Chair of Non-Destructive Testing and Quality Assurance, Saarland University, Am Markt Zeile 4, D-66125, \\ Saarbrücken, Germany. **haoran.wu@uni-saarland.de
}

\begin{abstract}
The fatigue behaviour of a metallic material can be characterized by extracting the material response during cyclic loading. Due to the good heat conductivity of metallic materials, thermographic NDT-methods are well suited to visualize and record the dissipated heat energy of specimens during cyclic loading, which is correlated mainly with the plastic deformation caused by the applied mechanical work. The acquired data can serve as a feed-in for short-time procedures or fatigue life calculation methods. In the framework of this paper, load increase and constant amplitude tests were performed and the cyclic deformation behaviour was traced by an infrared camera system.
\end{abstract}

\section{Introduction}

To describe the cyclic deformation behaviour of specimens subjected to fatigue loading, the plastic strain amplitude derived from measured mechanical stress-strain hysteresis is usually used. Beside conventional strain measurements, however, there are also other possibilities to describe the cyclic deformation behaviour and furthermore the damage state or evolution during a fatigue test. A specimen under fatigue loading basically goes through thermodynamic processes, which are characterized in a most general form by complex interactions between temperature, stress, strain and microstructure-sensitive material properties [1], e.g. the applied mechanical work during cyclic loading leads to energy dissipation and is transported by heat-transfer-processes to the specimen's surface, which can be measured as emitted heat. Due to this fact it is therefore useful to apply thermometric methods taking advantage of the specimen's change in temperature as an indicator for the fatigue behavior, analogous to the plastic strain amplitude [2].

Among the different thermometric methods [3-6], IR(infrared)-camera-based quantitative thermography has enormous advantages over other methods due to its non-contact feature and surface information to be visualized, which is therefore appropriate to provide more information regarding the fatigue behavior. Since Reifsnider and Williams applied an IR-camera in this field for the first time [7], there is an increasing number of publications dealing with similar methods [8-23]. Among these different activities having been published, the parameters commonly used to describe the change in temperature of defined measurement areas are $\Delta \theta[3,4,6], \Delta t[5]$ or $\Delta T$ [12], which are given different physical meanings by different authors while the symbols being similar. Fargione e.g. defines $\Delta T$ in [13] as the temperature difference between the starting and the stabilized state of a constant amplitude test (CAT). Shelp \& Eifler [24] and Starke et. al. [2,19,20] use $\Delta T$ to represent the temperature difference calculated by the formula $\Delta T=T_{1}-\left(T_{2}+T_{3}\right) / 2$, where $T_{1}$ is the surface temperature in the middle of the specimen's gauge length and $T_{2}$ as well as $T_{3}$ are the surface temperatures of both gauge length-shaft transitions having a significant thicker diameter, where only elastic deformations appear. Ummenhofer [11] derives a non-linear temperature amplitude $T_{a}^{\text {diss }}$ with NUC (non-uniformity-correction)-method. Meyendorf and Rösner $[21,25]$ subtract a reference temperature from the average temperature progress during fatigue loading and define $\Delta T_{\text {diss }}$ and its change for each cycle $\Delta \tau_{\text {diss }}$ as damage relevant indicators.

When taking thermodynamics during a fatigue process into account it seems to be more reasonable to analyse the temperature progress of the fatigue specimen considering the combination of the first and the second law of thermodynamics. Chrysochoos and Dupré have proposed their approach in [8], which was followed by work using dissipated energy to characterise fatigue processes [26-28]. Risitano et. al. have also done research by following the rule of conservation of energy and derived an integral $\Phi$ of the area below the change in temperature-time curve as the amount of liberated energy $Q$. It is assumed in that case that $\Phi$ stays constant for the same material and mechanical component respectively, no matter if the load or the change in temperature of one fatigue test changes [12-16].

With the analyses mentioned above, the fatigue limit (or fatigue strength) could be determined reliably and the fatigue life under cyclic loading could also be determined by a generated Wöhler (S-N) curve. Based on the PHYBAL- and Locati-method [12-17] which were introduced separately in the publications mentioned above, different stress-controlled constant amplitude tests and load increase tests (LITs) are carried out on specimens made of SAE 1045 steel (C45E: German designation), which lead to new interesting results, according to which the former assumptions are strongly challenged.

\section{Material and Specimen}

The fatigue specimens within the frame of this paper were machined identically from SAE 1045 steel, which is named after the German designation as C45E in accordance with DIN EN 10083-1. The material was delivered in form of round bars with a diameter of $20 \mathrm{~mm}$ and is characterized by a typical ferritic-pearlitic microstructure. The specimens are 
designed as an hourglass shape, as shown in figure 1, such that the middle along the gauge length has a diameter of $6 \mathrm{~mm}$, being half of the diameter of both of the shafts. More details regarding the specimen are given in [20].
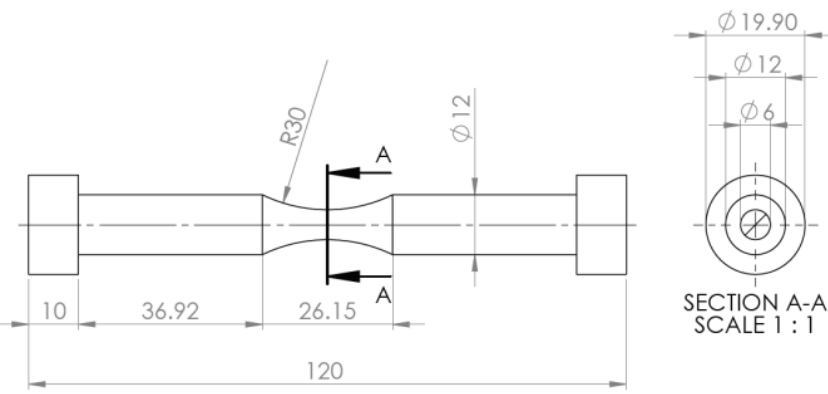

Fig. 1. Geometry of hourglass shaped specimen.

\section{Experimental setup}

For the observation of the fatigue specimen an infrared camera (Micro-Epsilon, type thermoIMAGER TIM450) was mounted in front of the specimen at a constant distance (figure 2), such that the Field of View (FOV) of each test has been kept the same. The IR-camera provides a spectral range of $7.5-13 \mu \mathrm{m}$ in wavelength, an optical resolution of 382 $\times 288$ pixels, a $29^{\circ}$ objective and a thermal sensitivity of $40 \mathrm{mK}$. Using the control software provided, it is easy to define the position and shape of the measurement areas on the specimen's surface to get the temperature data of $T_{1}, T_{2}$ and $T_{3}$ as mentioned in the introduction above. The recording frequency of the IR-camera was chosen at $f_{I R}=100 \mathrm{~Hz}$. Before a test starts, a thermal and IR-isolation is placed in order to reduce the influence of convection and radiation from the surrounding environment. Several thermocouples were attached to the clamping in the proximity of the specimen to monitor the ambient temperature (figure 3).

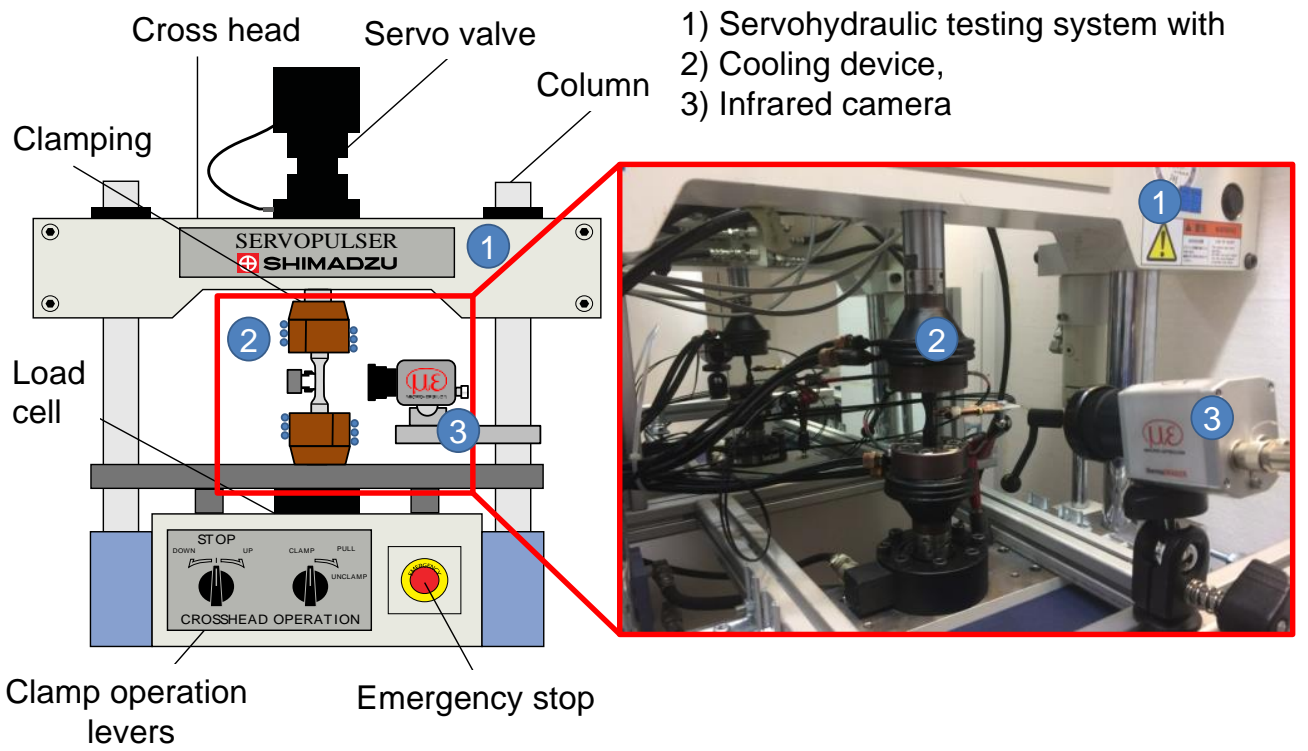

Fig. 2. Schematic view of the servo-hydraulic fatigue testing machine (left) and view on the matt-black-painted specimen mounted between the temperature-stabilized clamps with an infrared camera (right).

Stress-controlled CATs and LITs were carried out at room temperature on a Shimadzu servohydraulic testing system (figure 2) with a testing frequency $f_{\text {test }}=5 \mathrm{~Hz}$ and a maximum cyclic load of $\pm 20 \mathrm{kN}$. The load-time function is in this case sinusoidal at a load ratio of $R=-1$. A self-developed cooling device for both grips based on integrated thermoelectric coolers, a water circulation system and a control unit was designed, such that the thermal influence of the test machine can be compensated. Hence the temperature difference between the two ends of the specimen can be limited to $0.2 \mathrm{~K}$ or lower at the beginning of the test and no more than $1.0 \mathrm{~K}$ during the test. The fatigue tests were performed until failure or to a maximum number of cycles $N_{\max }=2 \cdot 10^{6}$. During the tests, temperature measurement areas on the specimen are defined with the same method as described in [18-20], where $T_{1}$ is in the middle of the specimen's gauge 
length, while $T_{2}$ and $T_{3}$ are 60 Pixels, corresponding approx. to a distance being $18.6 \mathrm{~mm}$ away from the point where $T_{1}$ has been measured (figure 3 ).

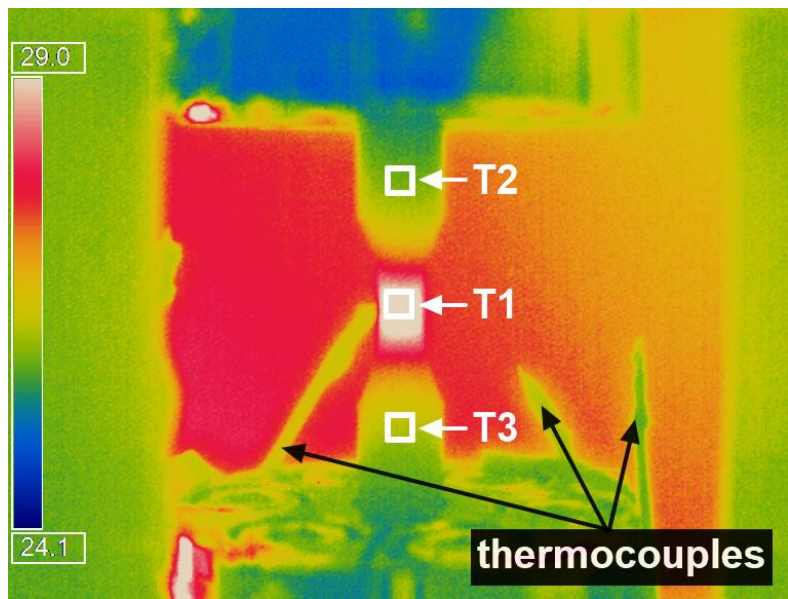

Fig. 3. Fatigue specimen during a test under the observation of an IR-camera, where thermocouples attached to the lower grip can be seen.

\section{Experimental tests and results}

Test data published in [18-20] have been used along with new test results, which were obtained from the same batch of specimens. These data were used to examine the former work of Risitano et. al. and Lipski [12-17]. 6 LITs (figure 4 left) were carried out with 5 different load increments $\left(\Delta \sigma_{a}\right)$ from 20 to $40 \mathrm{MPa}$ with identical step length $\Delta N$ at each load level. Each LIT begins at $\sigma_{a, s t a r t}=100 \mathrm{MPa}$, which is far below the evaluated fatigue limit. After $\Delta N=9,000 \mathrm{cycles}$, the stress amplitude is increased by a certain $\Delta \sigma_{a}$ value until failure. 8 CATs (figure 4 right) were carried out at 8 different stress amplitudes $\left(\sigma_{a}\right)$ ranging from 300 to $380 \mathrm{MPa}$ and were run up to failure.

The change in temperature, commonly used in the PHYBALLsv related experiments [18] and the SteBLife-method $[19,20]$, which is calculated by the formula $\Delta T=T_{1}-\left(T_{2}+T_{3}\right) / 2$, are further corrected by subtracting the very first value from every test, in order to make sure, that all the tests start with $\Delta T=0$.
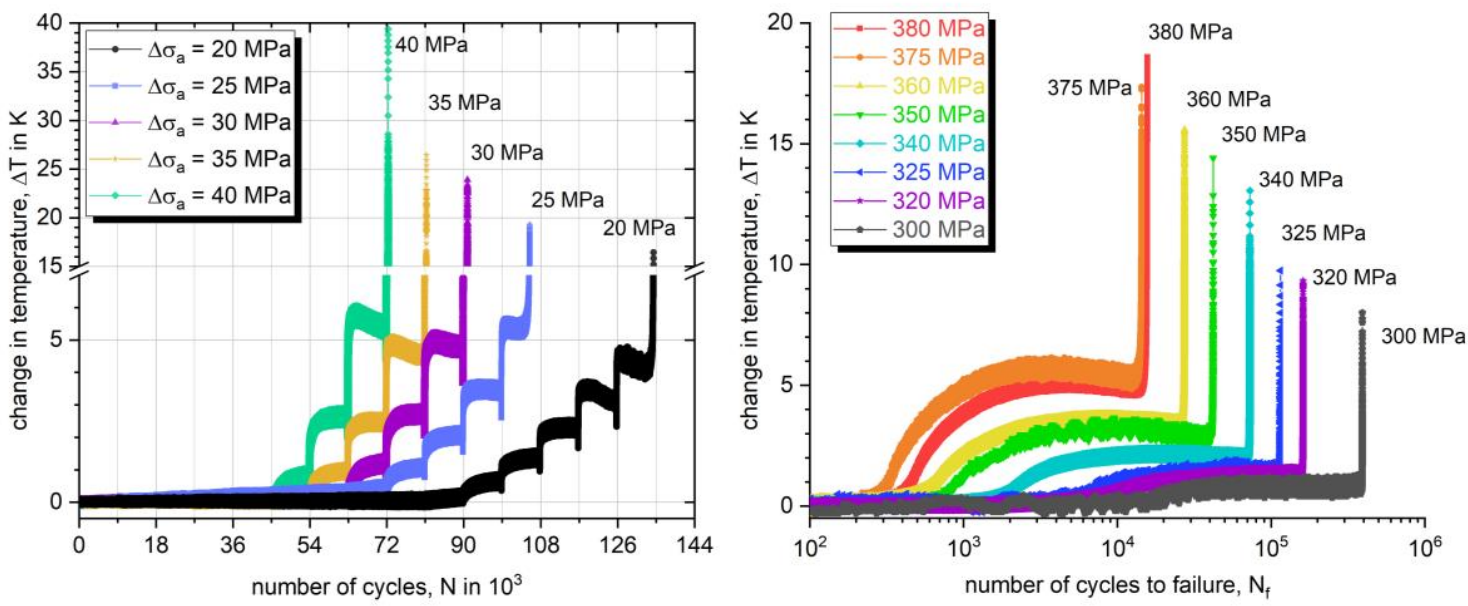

Fig. 4. Left: $\triangle T-N$ curves from LITs with different load increments, $y$-axis with broken. Right: $\Delta T-N$ curve from CATs with different stress amplitudes, $x$-axis logarithmic displayed. [18,19]

When not only taking the change in temperature into account, but also considering thermodynamics, it is meaningful to calculate the area of the region enclosing the $\Delta T-N$ curve, which represents the dissipated energy during the fatigue process $[17,29]$. It is emphasized, that this calculation is linked to the time $t$, load frequency $f_{\text {test }}$ and the IR-camera recording frequency $f_{I R}$. In reality, the $\Delta T-N$ curves shown above are affected by two more effects, which are caused separately by thermodynamics resulting from the elastic and non-elastic portion of cyclic loading. The first part, which is also called the thermoelastic- or Thomson-effect, was originally reported by William Thomson (Lord Kelvin) in 1853 [30] and describes the linear correlation between the change in temperature of a solid state material and the elastic 
loading applied. The change in temperature on a piece of material caused by thermoelasticity is considered to be governed by the stress on this piece, which can be mathematically described as:

$$
\Delta T_{e l}=-\frac{\alpha}{\rho c} \cdot T_{0} \cdot \sigma_{a}
$$

where the parameters represent the following:

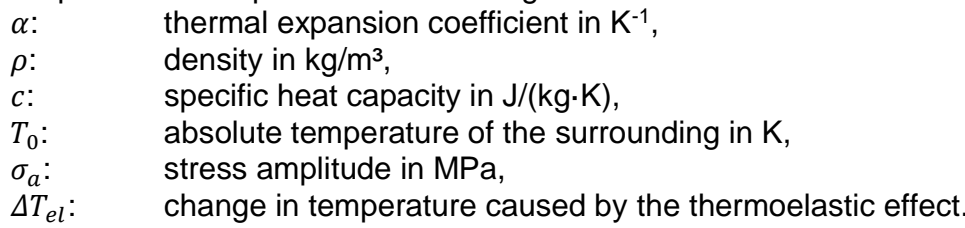

Assuming that the stress amplitude-time curve is sinusoidal, the resulting temperature change follows a sinusoidal function as well and can be written as:

$$
\Delta T_{e l}(t)=-A \cdot \sin (\omega t+\varphi)
$$

where $\omega$ represents the angular velocity, $\varphi$ the phase change and $A$ the right hand term of Eq. (1) as the amplitude respectively. For a complete test it can be assumed that

$$
\begin{gathered}
\omega=2 \pi \cdot f_{\text {test }} \\
\varphi=0 \\
\text { and } \\
N_{f}=t \cdot f_{\text {test }}=i \cdot \frac{f_{\text {test }}}{f_{I R}}
\end{gathered}
$$

where $N_{f}$ is the number of cycles to failure, $f_{\text {test }}$ the cycling frequency, $f_{I R}$ the sampling rate of the temperature measurement and $i$ the number of data recorded by the IR-camera. During cycling of the material two thermal parameters can be determine, one resulting from elasticity only and defined by Eq. (6) and one resulting from non-elasticity defined by Eq. (7) below:

$$
\begin{gathered}
\tau_{e l}=N_{f} \cdot \int_{0}^{2 \pi} \Delta T_{e l}(t) \cdot d t=N_{f} \cdot \int_{0}^{2 \pi}-A \cdot \sin \left(2 \pi \cdot f_{\text {test }} \cdot t\right) \cdot d t=0 \\
\tau_{\text {non-el }}=\int_{0}^{N_{f}} \Delta T(N) \cdot d N=\frac{f_{\text {test }}}{f_{I R}} \cdot \int_{0}^{i} \Delta T(i) \cdot d i=\frac{f_{\text {test }}}{f_{I R}} \cdot \sum_{0}^{i} \Delta T(i)
\end{gathered}
$$

While the elasticity related term in Eq. (6) becomes comparatively small and is principally zero in case a material behaves the same under tensile and compressive loading, the non-elasticity related parameter can become significant. Instead of integrating over time, an integration over the number of cycles is also valid, specifically when loading is performed under a constant frequency. For a constant amplitude test and a frequency being recorded, the total integral of change in temperature $\tau$ (Greek letter tau) is defined as a summary of the elastic and the non-elastic parameter as:

$$
\tau=\tau_{e l}+\tau_{\text {non-el }} \approx \sum_{0}^{i} \Delta T(i)
$$

which can also be simplified as a summation of the temperature change per measurement each. From the Eqs. (7) and (8), Eq. (8.1) for all CATs and Eq. (8.2) for each single step of the LITs can be derived as follow:

$$
\tau_{C A T}=\int_{0}^{N_{f}} \Delta T(N) \cdot d N \approx \sum_{0}^{i} \Delta T(i) \quad, \text { for } i=\frac{f_{\text {test }}}{f_{I R}} \cdot N_{f}
$$

and for LITs on the every single $j$-th load step:

$$
\tau_{L I T}=\int_{(j-1) \cdot \Delta N}^{j \cdot \Delta N} \Delta T(N) \cdot d N \approx \sum_{(j-1) \cdot i}^{j \cdot i} \Delta T(i) \quad \text {, for } j=1,2,3, \ldots, n
$$

where the upper limit of the summation $j \cdot i$ equals $\frac{f_{\text {test }}}{f_{I R}} \cdot j \cdot \Delta N$, if the specimen runs through a complete load step. Otherwise it would be $\frac{f_{\text {test }}}{f_{I R}} \cdot\left(N_{f}-(j-1) \cdot \Delta N\right)$, if the specimen breaks during the test on the $j$-th load step. For all the tests performed the results can be described in accordance to Eqs. (8.1) and (8.2). This was undertaken for all CATs and separately for each single step of the LITs. In order to compare the results of CATs and LITs, results of stress levels from $300 \mathrm{MPa}$ on of the LITs with $\Delta \sigma_{a}=20 \mathrm{MPa}, 25 \mathrm{MPa}$ and $40 \mathrm{MPa}$ were displayed in figure 5 where corresponding values of stress amplitudes have been available. 


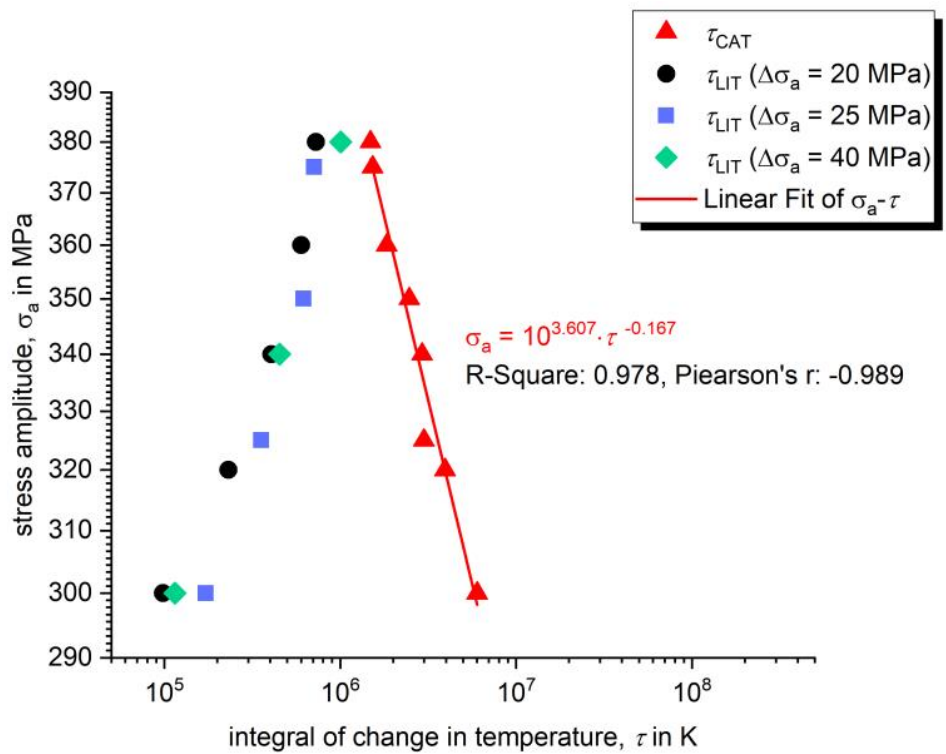

Fig. 5. Stress amplitude versus the integral of change in temperature of CATs (complete test, red points) and LITs (each load steps from 300 MPa on, black, green and blue points), double logarithmic displayed.

From the results obtained here it can be seen that $\tau$ changes with the stress level applied for the different CATs and that this can be linearized when being plotted in a logarithmic scale for $\tau$, resulting in $\sigma_{a}=103.607 \cdot \tau^{-0.167}$, with a $\mathrm{R}$-square value of 0.978 for the present case. This is in contrast to what has been stated in [29] so far, where the value of $\tau$ has been assumed constant. However, for LITs this trend when plotted in accordance to each stress amplitude level is and should be inverse to that of CAT, being a proof of a material's non-linear damage accumulation, specifically in the high cycle fatigue (HCF) regime, where those tests are supposed to be. A further question arises as to what the point represents at which the two trend lines of the CATs and the LITs meet. This looks to be at or around the material's yield strength which even for an un-notched specimen would mean, that the minimum section of the specimen would have generally passed the yield strength while below this stress level this minimum section would only be partially plastified. The portions of plastification therefore have to differ between a CAT and a LIT as a consequence of the loading sequence applied.

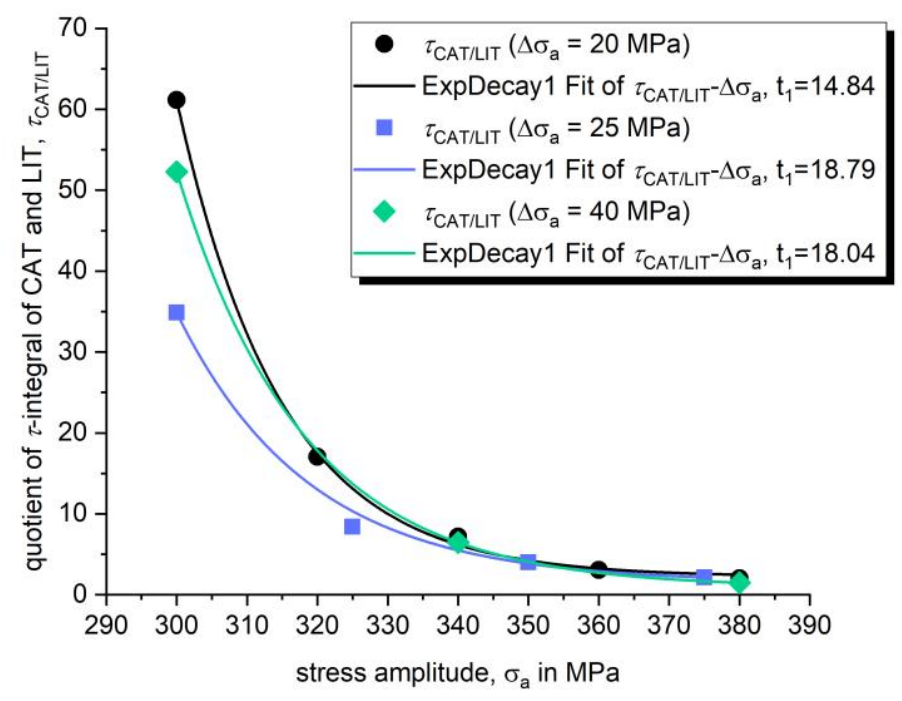

Fig. 6. Ratios of $\tau$-integrals from CAT and LIT $\tau_{C A T / L I T}$ versus stress amplitude $\sigma_{a}$ and resulting fits.

When plotting the ratio of $\tau$-values between CAT and LIT over the stress amplitudes being applied as shown in figure 6 an exponential decay relationship is applied and can be described by Eq. (9) below:

$$
\tau_{C A T / L I T}=\tau_{C A T / L I T_{\text {min }}}+\left(\tau_{C A T / L I T_{\text {max }}}-\tau_{C A T / L I T} \text { min }\right) \cdot \exp \left(\left(\sigma_{a_{\min }}-\sigma_{a}\right) / t_{1}\right)
$$


where $\tau_{C A T / L I T}$ is the ratio of $\tau$-values between CAT and LIT, the maximum or minimum value of which are followed with the subscript max or min; $\sigma_{a}$ the stress amplitude, whose minimum value is in this case $300 \mathrm{MPa} ; t_{1}$ the time constant that describes the degree of the decay.

This relationship does not significantly differ between the different stress amplitude steps applied with the different LITs and can be used to convert LIT based results to CATs, showing that this is not truly a linear relationship as assumed in the past but rather a non-linear one that may be considered when determining S-N curves as described in [2] and [18] respectively.

In [17] a relationship has been mentioned as expressed in Eq. (10) below

$$
\frac{\varphi_{i}}{\Phi_{i}}=\frac{n_{i}}{N_{i}}
$$

where $\varphi_{i}$ represents the integral of change in temperature of a single load step from the LIT, $\Phi_{i}$ the corresponding integral from the CAT with the same stress amplitude, $n_{i}$ the step length of the LIT and $N_{i}$ the fatigue life of the corresponding CAT. If this relationship is to be expressed with the terms used above, Eq. (10) turns to be

$$
\frac{\tau_{L I T}}{\tau_{C A T}}=\frac{\Delta N}{N_{f}}
$$

The left hand part of Eq. (11) is nothing else than the inversion of the ratio of the $\tau$-integral provided in figure 6 . With respect to the LITS, each of the points indicated for a LIT represents the damage contribution of each of the loading steps. These contributions of damage are indicated as a summary in the left half of figure 7 and show that the results vary around a value of $100 \%$. When a classical Palmgren-Miner fatigue life estimation is done with the S-N data obtained, then the right hand portion of Eq. (11) is obtained and it can be observed, that the trends but not the numbers are the same (right half of figure 7). Hence the equality assumed in Eqs. (10) as well as (11) cannot be considered to be proven but rather looks to be a simplification of a more complex process in which the $\tau$-integral approach might allow to provide some more tangible results, still not determined today.

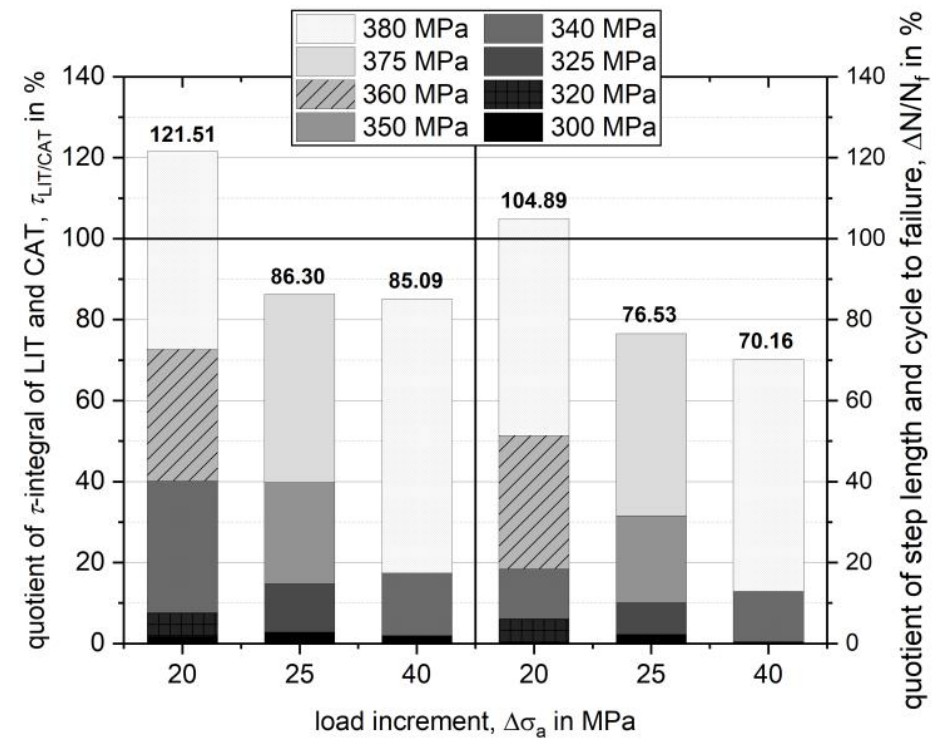

Fig. 7. Comparison between the damage accumulation assumption by using $\tau$-integral and number of cycles (left half) and conventional Palmgren-Miner based fatigue life evaluation (right half) for different load increase tests with different load increment.

\section{Conclusions and discussion}

Quantitative thermography has been increasingly used in the last decades to evaluate the fatigue behaviour of materials for different purposes. By integrating a measured temperature range $\Delta T$ over the number of loading cycles $N$ a new parameter named the $\tau$-integral is proposed that allows damage and hence damage accumulation in a material to be quantified in a more appropriate way. A relationship between the $\tau$-integral and the number of cycles to failure $N_{f}$ can be expressed through a logarithmic function. This has been shown for a SAE 1045 steel with a high degree of confidence. Although the $\tau$-integral has been known before from literature it has been considered as a constant value independent of the stress amplitude applied. However, the data being presented here do show a logarithmic dependence and therefore the assumption of a constant value may not hold. Correlating the $\tau$-integral values between CATs and LITs for identical stress amplitude levels as a ratio provides an interesting indicator as regards the damage contribution of each of a LIT's stress levels and could possibly become an indicator as to a material's non-linear damage accumulation 
behaviour. Furthermore it could be used to further improve the process when the results from a LIT are used to determine the behaviour of CATs in terms of an S-N curve estimation.

The approach presented here needs further validation in many regards with respect to higher stress levels or different materials. The approach also needs further validation in terms of the influence of arbitrary load sequences, specifically the effect of load sequence effects and how far those can be quantifiably monitored and described as well. Similar trials may also be useful with regard to the determination of the endurance limit. With this not only a new damage parameter is created but also a new scope along which the non-linear accumulation of damage could be grasped in a more quantifiable way. Due to the integral way of monitoring by thermography the information retrieved may not be limited to a single point only but may allow larger areas to be scanned, hopefully in the not too distant future.

\section{ACKNOWLEDGEMENT}

The authors would like to thank the German Research Foundation (Deutsche Forschungsgemeinschaft DFG) for the financial support of this research (STA 1133/6-1). The authors would also thank Shimadzu Europe GmbH and Micro-Epsilon Messtechnik $\mathrm{GmbH} \& \mathrm{Co}$. KG for their support in technical equipment provision.

\section{REFERENCES}

[1] Altenbach, H; Altenbach, J; Münch C. Analyse des thermomechanischen Verhaltens von Festkörpern. Arch Appl Mech 1991;61:245-58.

[2] Starke P, Walther F, Eifler D. "PHYBAL" - Short-time calculation of Woehler curves of wheel steels. ZEV Rail 2009;133:251-5.

[3] Dillon OW. An experimental study of the heat generated during torsional oscillations. J Mech Phys Solids 1962;10:235-44. doi:10.1016/0022-5096(62)90040-6.

[4] Dillon OW. Coupled thermoplasticity. J Mech Phys Solids 1963;11:21-33. doi:10.1016/0022-5096(63)90004-8.

[5] Dengel D, Harig H. Estimation of the fatigue limit by progressively - incresing load tests. Fatigue Eng Matérials Struct 1980;3:113-28.

[6] Stärk KF. Temperaturmessung an schwingend beanspruchuten Werkstoffen. Z Werkstofftech 1982;13:333-8.

[7] Reifsnider KL, Williams RS. Determination of fatigue-related heat emission in composite materials. Exp Mech 1974;14:479-85. doi:https://doi.org/10.1007/BF02323148.

[8] Chrysochoos A, Dupre JC. An infrared set-up for continuum thermomechanics. QIRT, vol. 27, Paris: 1992. doi:http://dx.doi.org/10.21611/qirt.1992.019.

[9] Luong MP. Infrared thermographic scanning of fatigue in metals. Nucl Eng Des 1995;158:363-76. doi:10.1016/0029-5493(95)01043-H.

[10] Luong MP. Fatigue limit evaluation of metals using an infrared thermographic technique. Mech Mater 1998;28:155-63.

[11] Ummenhofer T, Medgenberg J. On the use of infrared thermography for the analysis of fatigue damage processes in welded joints. Int J Fatigue 2009;31:130-7. doi:10.1016/j.ijfatigue.2008.04.005.

[12] La Rosa G, Risitano A. Thermographic methodology for rapid determination of the fatigue limit of materials and mechanical components. Int J Fatigue 2000;22:65-73.

[13] Fargione G, Geraci A, La Rosa G, Risitano A. Rapid determination of the fatigue curve by the thermographic method. Int J Fatigue 2002;24:11-9.

[14] Risitano A, Risitano G. Cumulative damage evaluation of steel using infrared thermography. Theor Appl Fract Mech 2010;54:82-90. doi:10.1016/j.tafmec.2010.10.002.

[15] Risitano A, Risitano G. Cumulative damage evaluation in multiple cycle fatigue tests taking into account energy parameters. Int J Fatigue 2013;48:214-22. doi:10.1016/j.ijfatigue.2012.10.020.

[16] Risitano A, Fargione G, Giudice F, Patanè G. Evaluation of the Relative Plastic Work Factor during the Fatigue Test. Procedia Eng 2015;109:346-55. doi:10.1016/j.proeng.2015.06.242.

[17] Lipski A. Determination of Fatigue Limit by Locati Methodusing S-N Curve Determined by Means of Thermographic Method. Solid State Phenom 2014;223:362-73. doi:10.4028/www.scientific.net/SSP.223.362.

[18] Starke, P; Wu, H.; Boller C. Advanced Evaluation of Fatigue Phenomena Using Non-Destructive Testing Methods. In: Sommitsch C, Ionescu M, Mishra B, Kozeschnik E, Chandra T, editors. 9th Int. Conf. Process. Manuf. Adv. Mater. THERMEC 2016, Graz, Austria: Trans Tech Publications; 2016, p. $1841-6$. doi:10.4028/www.scientific.net/MSF.879.1841.

[19] Starke P, Bäumchen A, Wu H. SteBLife - A new short-time procedure for the calculation of S-N curves and failure probabilities. Mater Test 2018;60:1-7. doi:10.3139/120.111139.

[20] Starke P, Wu H. Use of non-destructive testing methods in a new one-specimen test strategy for the estimation of fatigue data. Int J Fatigue 2018;111:177-85. doi:10.1016/j.ijfatigue.2018.02.011.

[21] Meyendorf NGH, Rösner H, Kramb V, Sathish S. Thermo-acoustic fatigue characterization. Ultrasonics 2002;40:427-34. doi:10.1016/S0041-624X(02)00155-5. 
[22] Ezanno A, Doudard C, Calloch S, Heuzé JL. A new approach to characterizing and modeling the high cycle fatigue properties of cast materials based on self-heating measurements under cyclic loadings. Int J Fatigue 2013;47:23243. doi:10.1016/j.ijfatigue.2012.09.005.

[23] Amiri M, Naderi M, Khonsari MM. An experimental approach to evaluate the critical damage. Int J Damage Mech 2011;20:89-112. doi:10.1177/1056789509343082.

[24] Schelp M, Eifler D. Evaluation of the HCF-behavior of 42CrMoS4 by means of strain, temperature and electrical measurements. Mater Sci Eng A 2001;319-321:652-6. doi:10.1016/S0921-5093(01)00981-9.

[25] Rösner H. THermographic Nondestructive Evalutation of Early Stages Fatigue Damage in Titanium Material. Universität des Saarlandes, 2003.

[26] Boulanger T, Chrysochoos A, Mabru C, Galtier A. Calorimetric analysis of dissipative and thermoelastic effects associated with the fatigue behavior of steels. Int J Fatigue 2004;26:221-9.

[27] Berthel B, Wattrisse B, Chrysochoos A, Galtier A. Thermographic analysis of fatigue dissipation properties of steel sheets. Strain 2007;43:273-9. doi:10.1111/j.1475-1305.2007.00349.x.

[28] Pastor ML, Balandraud X, Grédiac M, Robert JL. Applying infrared thermography to study the heating of 2024-T3 aluminium specimens under fatigue loading. Infrared Phys Technol 2008;51:505-15. doi:10.1016/j.infrared.2008.01.001.

[29] Fargione G, Geraci A, La Rosa G, Risitano A. Rapid determination of the fatigue curve by the thermographic method. Int J Fatigue 2002;24:11-9. doi:10.1016/S0142-1123(01)00107-4.

[30] Thomson W. Dynamical theory of heat with numerical results deduced from Mr Joule's equivalent of a thermal unit and Mr Regnault's observation on steam. Trans R Soc Edinburgh 1853;20:261-83. 